\title{
Next-to-leading order simulations with Sherpa+OpenLoops
}

\author{
Fabio Cascioli, ${ }^{a}$ Stefan Höche,${ }^{b}$ Frank Krauss, ${ }^{c}$ Philipp Maierhöfer ${ }^{*}{ }^{a}$ \\ Niccolò Moretti, ${ }^{a}$ Stefano Pozzorini, ${ }^{a}$ Marek Schönherr ${ }^{c}$ and Frank Siegert ${ }^{d}$ \\ ${ }^{a}$ Institut für Theoretische Physik, Universität Zürich, 8057 Zürich, Switzerland \\ ${ }^{b}$ SLAC National Accelerator Laboratory, Menlo Park, CA 94025, USA \\ ${ }^{c}$ Institute for Particle Physics Phenomenology, Durham University, Durham DH1 3LE, UK \\ ${ }^{d}$ Institut für Kern- und Teilchenphysik, TU Dresden, D-01062 Dresden, Germany \\ E-mail: cascioli@physik.uzh.ch, shoecheeslac.stanford.edu, \\ frank.kraussedurham.ac.uk, philipp@physik.uzh.ch, \\ moretti@physik.uzh.ch, pozzorin@physik.uzh.ch, \\ marek.schoenherrddurham.ac.uk, frank.siegertecern.ch
}

\begin{abstract}
We report on the SHERPA+OPENLOOPS framework for fully automated simulations of scattering processes at next-to-leading order. OPENLOOPS performs the fast evaluation of one-loop matrix elements and is interfaced to the SHERPA Monte Carlo event generator. The framework is applied

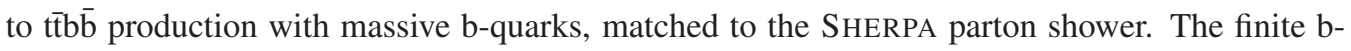
quark mass allows us to extend the kinematics to collinear b-quarks from $\mathrm{g} \rightarrow \mathrm{b} \overline{\mathrm{b}}$ splittings. We find that this splitting mechanism has a significant impact in the $\mathrm{t} \overline{\mathrm{t}} \mathrm{H}(\mathrm{b} \overline{\mathrm{b}})$ signal region. Furthermore we address tt production in conjunction with up to two jets merged with the MEPs@NLO method, resulting in a unified description of top-pair plus multi-jet production.
\end{abstract}

Loops and Legs in Quantum Field Theory - LL 2014,

27 April - 2 May 2014

Weimar, Germany

* Speaker. 


\section{Introduction}

In the last few years the feasibility of next-to-leading order (NLO) QCD corrections to $2 \rightarrow 4$ scattering amplitudes has been well established. Several programs have been developed to automate as far as possible the generation of NLO matrix elements for arbitrary multi-leg processes [1 -5]. In particular, the OPENLOOPS program [1], which is based on the open-loops algorithm for the recursive construction of loop momentum polynomials for Feynman diagrams, allows for the highly efficient evaluation of tree and one-loop matrix elements. The algorithm was inspired by a DysonSchwinger-like recursion introduced in the context of multi-gluon scattering [6]. OPENLOOPS uses the COLLIER library for the numerically stable evaluation of scalar and tensor integrals [79]. In Sherpa+Openloops we combined Openloops with the Sherpa Monte Carlo event generator [10] to fully automate NLO simulations which are suitable for experimental analyses. SHERPA provides the real-emission contributions, infrared subtraction, phase-space integration, S-MC@NLO matching [11, 12] (the SHERPA formulation of MC@ NLO matching [13]) to the SHERPA parton shower, and MEPs @ NLO multi-jet merging [14, 15].

Within this framework we calculated QCD corrections to top-quark pair production in association with a pair of massive bottom quarks, matched to the parton shower [16], and top-quark pair production in association with two jets, merged with lower jet multiplicities with the MEPs@ NLO method [17]. In the following two sections, selected results of these two projects are presented, followed by our conclusions.

\section{2. $\mathbf{t} \overline{\mathrm{t}} \mathrm{b} \overline{\mathrm{b}}$ with massive b-quarks matched to a parton shower}

Higgs-boson production in association with a top-quark pair, where the Higgs-boson decays into bottom-quarks, provides the opportunity to test the mass-generation mechanism in the heavyquark sector. However, the $\bar{t} \bar{H}$ signal is strongly contaminated by contributions from top-quark pair production and lighter quark pairs, making the Higgs mass peak difficult to identify. NLO calculations for $\bar{t} \overline{\mathrm{t}} \mathrm{b}$ production [18-21] reduce perturbative uncertainties considerably, however, in order to be applicable to the experimental analyses, these calculations need to be matched to parton showers. First technical results towards NLO matched tt̄b $\bar{b}$ production have been discussed in [22].

Here we discuss a NLO simulation of t⿱亠䒑十b $\bar{b}$ production matched to the parton shower with the

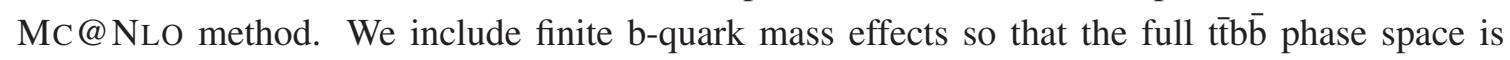
accessible, thereby including also $t \bar{t}+1 b$-jet contributions with an unresolved (soft or collinear) $b$ quark, which play an important role in the $t \bar{t} \mathrm{H}(\mathrm{b} \bar{b})$ analysis. Moreover, in configurations where the two b-jets both arise from collinear $g \rightarrow b \bar{b}$ splittings, the finite $b$-quark mass allows us to describe one of the splittings at NLO accuracy instead of relying only on parton shower splittings on top of ț̈gg matrix elements.

We compare leading order (LO), NLO and MC@ NLO simulations of ttib $\bar{b}$ production at the $8 \mathrm{TeV}$ LHC. Top quarks are treated as stable particles with mass $m_{\mathrm{t}}=173.2 \mathrm{GeV}$ to focus on the behaviour of those b-jets which arise from QCD interactions. Consistently with the finite b-quark mass, $m_{\mathrm{b}}=4.75 \mathrm{GeV}$, we employ four-flavour MSTW2008NLO (LO) parton distributions [23] at NLO (LO) QCD. As renormalisation scale we use the geometric average of the top-quark and b- 

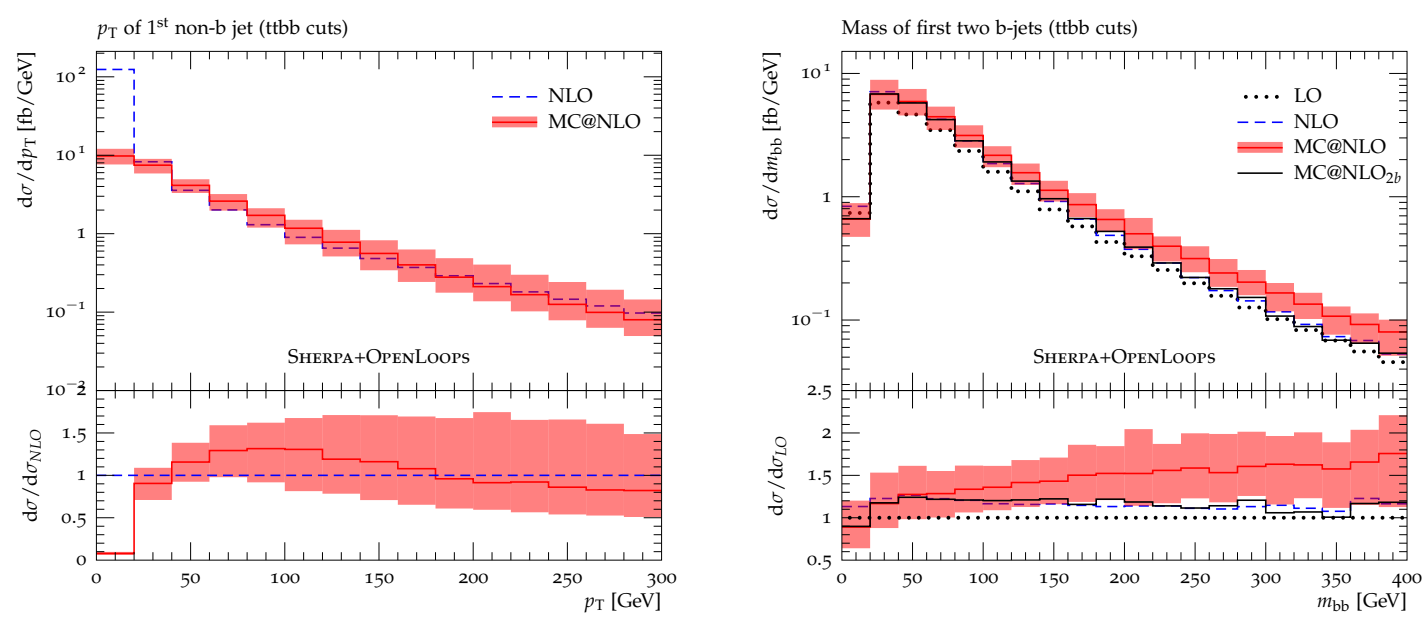

Figure 1: Transverse momentum of the first light jet (left) and invariant mass of the first two b-jets (right) with standard ttbb cuts. The MC @ NLO bands display the combination in quadrature of $\mu_{\mathrm{R}}, \mu_{\mathrm{F}}$ and $\mu_{\mathrm{Q}}$ scale variations. The MC@ $\mathrm{NLO}_{2 b}$ curve is obtained by switching off $\mathrm{g} \rightarrow \mathrm{b} \overline{\mathrm{b}}$ splittings in the parton shower.

quark transverse energies, $\mu_{\mathrm{R}}^{4}=\prod_{i=\mathrm{t}, \overline{\mathrm{t}}, \mathrm{b}, \overline{\mathrm{b}}} E_{\mathrm{T}, i}$ with $E_{\mathrm{T}, i}=\sqrt{m_{i}^{2}+p_{\mathrm{T}, i}^{2}}$, so that the strong coupling factors adapt to the transverse energies of the final-state particles. The factorisation and resummation scales, $\mu_{\mathrm{F}}$ and $\mu_{\mathrm{Q}}$, are set to the average top-quark transverse energy $\mu_{\mathrm{F}}=\mu_{\mathrm{Q}}=\frac{1}{2}\left(E_{\mathrm{T}, \mathrm{t}}+E_{\mathrm{T}, \overline{\mathrm{t}}}\right)$. QCD partons, including b-quarks and excluding only top-quarks, are recombined into anti- $k_{\mathrm{T}}$ jets [24] with $R=0.4$. Events are categorised according to the number $N_{\mathrm{b}}$ of reconstructed b-jets with $p_{\mathrm{T}}>25 \mathrm{GeV}$ and $\left|\eta_{\mathrm{b}}\right|<2.5$. As a realistic definition of b-jets, any jet which contains at least one b-quark is classified as such. To investigate NLO and MC@ NLO correction effects we consider a ttbb sample with events involving $N_{\mathrm{b}} \geq 2 \mathrm{~b}$-jets. A further analysis is performed with a cut on the invariant mass of the first and second b-jet, $m_{\mathrm{bb}}>100 \mathrm{GeV}$, which corresponds to the Higgs signal region. Scale uncertainties are assessed via factor 2 variations of $\mu_{\mathrm{R}}$ and $\mu_{\mathrm{F}}$ and factor $\sqrt{2}$ variations of the resummation scale. Scale uncertainties are dominated by renormalisation-scale variations and decrease from about $60-70 \%$ at LO to 20-30\% at NLO rsp. Mc @ NLO.

Fig. 1 (left) shows the transverse momentum of the first non-b-jet, which exhibits the typical Mc@ NLo behaviour. At transverse momenta above the resummation scale, Mc@ NLO and NLO predictions agree well. The fixed-order infrared singularity at small $p_{\mathrm{T}}$ is consistently damped by the Sudakov form factor, and Sudakov effects start to be important already at $p_{\mathrm{T}} \sim 50 \mathrm{GeV}$. This reflects the presence of intense QCD radiation resulting from the gluon-gluon initial state and from the high centre-of-mass energy of the t⿱ẗb $\bar{b}$ system. The invariant mass distribution of the two b-jets depicted in Fig. 1 (right) shows that matching corrections are quite sensitive to the invariant mass of the first two b-jets. The Mc @ NLO/NLO ratio grows with $m_{\mathrm{bb}}$ and reaches $25-30 \%$ in the region $m_{\mathrm{bb}} \sim 125 \mathrm{GeV}$, exceeding the expected Higgs signal. To reveal the origin of this effect, we show that in an (unphysical) approximation with $g \rightarrow b \bar{b}$ splittings switched off in the parton shower, the excess disappears, indicating that it is dominated by double gluon splittings. This mechanism is kinematically favoured by the fact that the probability that two hard gluons split into collinear $b \bar{b}$ pairs does not decrease when the invariant mass of the gluon pair grows. Further evidence of the 

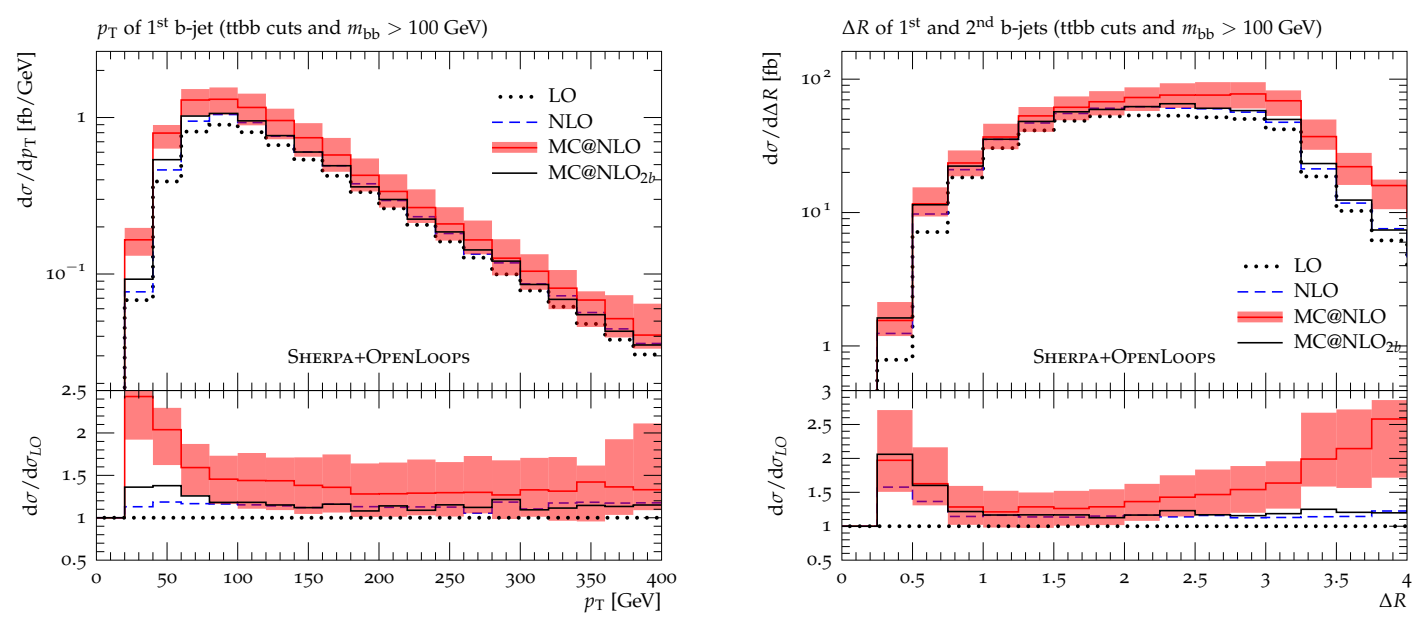

Figure 2: Transverse momentum of the first b-jet and $\Delta R$ separation of the first two b-jets with standard ttbb cuts and $M_{\mathrm{bb}}>100 \mathrm{GeV}$. See Fig. 1 for details.

correctness of the above picture is provided by the fact that the MC@ NLO excess increases with

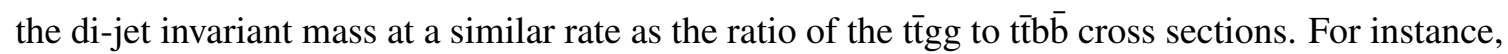
using LO matrix elements, we checked that both quantities increase by a factor two in the range between 100 and $250 \mathrm{GeV}$.

The plots in Fig. 2, where an additional cut $m_{\mathrm{bb}}>100 \mathrm{GeV}$ is applied, reveal distinctive kinematic features of the Mc@ NLO enhancement in the Higgs-signal region. The Mc@NLO/NLO peaks that appear in the distributions, both in the transverse momentum of the first b-jet (left) and in the $\Delta R$ separation of the first two b-jets (right), show that the MC@ NLO enhancement is dominated by back-to-back b-jets with the smallest possible $p_{\mathrm{T}}$ that is needed to reach $m_{\mathrm{bb}}=100 \mathrm{GeV}$. This is consistent with the expected behaviour of double $g \rightarrow b \bar{b}$ splitting contributions, where emissions at small $p_{\mathrm{T}}$ are doubly enhanced by soft and collinear singularities associated with the parent gluons.

\section{3. tit production merged with up to two jets at NLO}

At the leading order in perturbative QCD, top-quark pair production at hadron colliders suffers from large theoretical uncertainties, which grow rapidly with the number of additional jets. Several calculations were completed recently to reduce these uncertainties. The inclusive production cross section has been determined at next-to-next-to leading order [25], and parton-level predictions of top-quark pair production in association with up to two jets have been computed at next-to leading order [26-28], and NLO calculations with one jet were matched to parton showers [29, 30] and merged with 0 -jet matrix elements [31, 32].

We use SHERPA+OPENLOOPS to match NLO matrix elements for the production of top-quark pairs in association with up to two jets to the parton shower and merge with lower jet multiplicities using the MEPs@NLO method to simultaneously describe $t \bar{t}+0,1,2$ jet configurations at NLO plus parton shower accuracy. The results are compared to two different simulations of lower ac- 

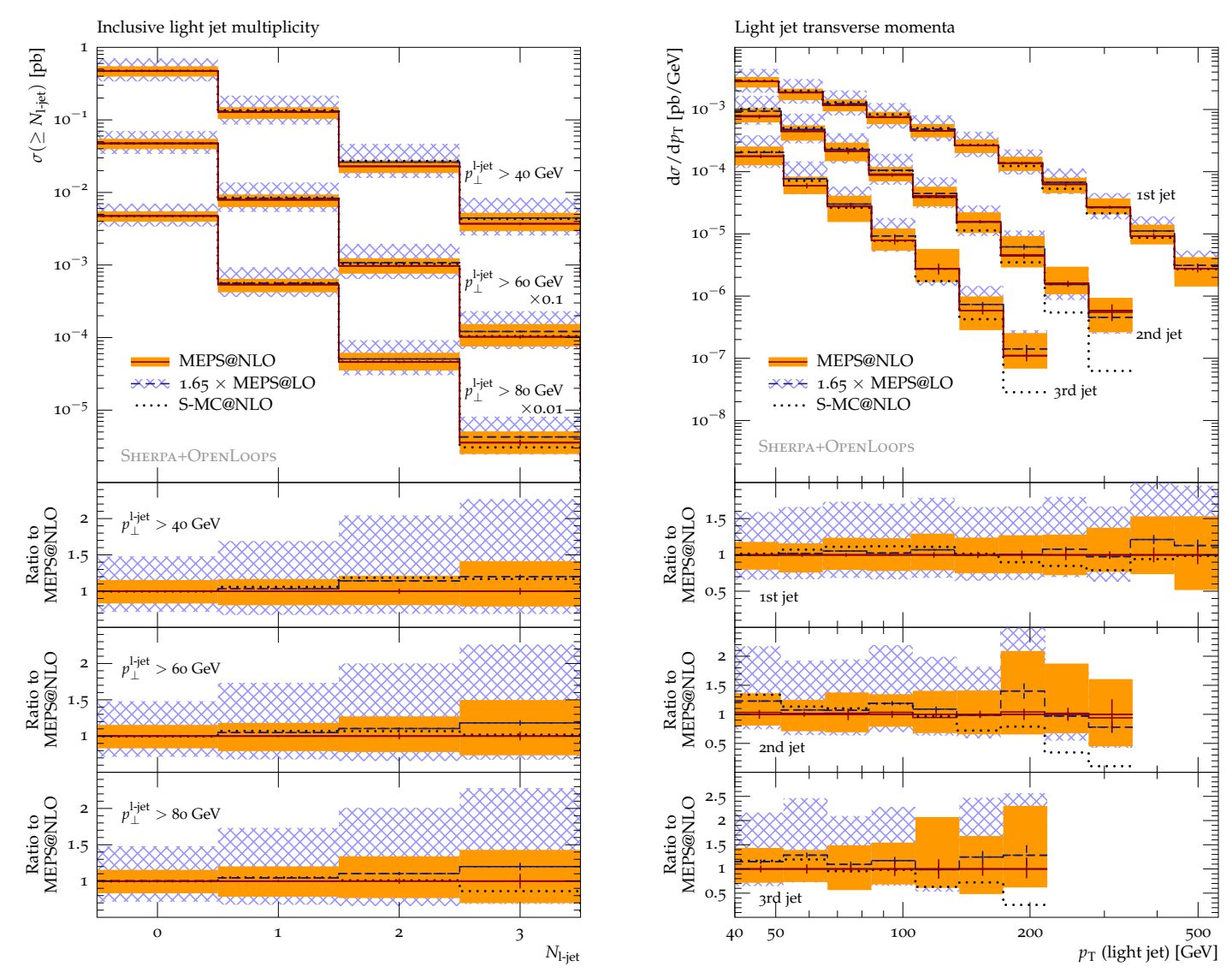

Figure 3: Light-flavour jet multiplicity distribution (including c- but not b-jets) for transverse momentum thresholds of 40,60 and $80 \mathrm{GeV}$ (left) and transverse momentum spectra of the three leading light-flavour jets (right). Solid (red) lines indicate MEPs@ NLO predictions, and the full (orange) band shows the corresponding total theoretical uncertainty. Dashed lines indicate MEPs@Lo predictions, with the corresponding uncertainties shown as hatched (blue) bands. S-Mc@ NLO predictions are shown as dotted histograms. Statistical uncertainties for each calculation are indicated by error bars.

curacy: MEPs @LO merging with a NLO $k$-factor, which is currently the default accuracy used in many experimental analyses, and S-MC@ NLO matching of tt to the parton shower.

The simulations are for the 7 TeV LHC using the MSTW2008NLO PDF set and include spin correlated top-quark decays at leading order. The renormalisation scale is defined via $\alpha_{\mathrm{s}}\left(\mu_{\mathrm{R}}\right)^{2+n}=$ $\alpha_{\mathrm{s}}\left(\mu_{\text {core }}\right)^{2} \Pi \alpha_{\mathrm{s}}\left(t_{i}\right)$ with the core scale $1 / \mu_{\text {core }}^{2}=1 / s+1 /\left(m_{t}^{2}-t\right)+1 /\left(m_{t}^{2}-u\right)$ and the clustering scales $t_{i}$ which correspond to the jet emissions. $\mu_{\text {core }}$ is also used as factorisation scale $\mu_{\mathrm{F}}$ and as the parton-shower starting scale $\mu_{\mathrm{Q}}$. The merging scale is set to $Q_{\text {cut }}=30 \mathrm{GeV}$. Theoretical uncertainties are estimated by factor 2 variations of $\mu_{\mathrm{R}}$ and $\mu_{\mathrm{F}}$, factor $\sqrt{2}$ variations of $\mu_{\mathrm{Q}}$, and $Q_{\mathrm{cut}}$ is varied between 20 and $40 \mathrm{GeV}$. Parton shower uncertainties are assessed by variation between the two recoil schemes described in [33, 34]. For further details about the analysis we refer to [17].

Fig. 3 shows the light-flavour jet multiplicity distribution for various transverse momentum thresholds (left) and the transverse momentum spectra of the three leading light-flavour jets (right). The MEPs@NLO results, which are our most accurate predictions, are compared to MEPs@LO 

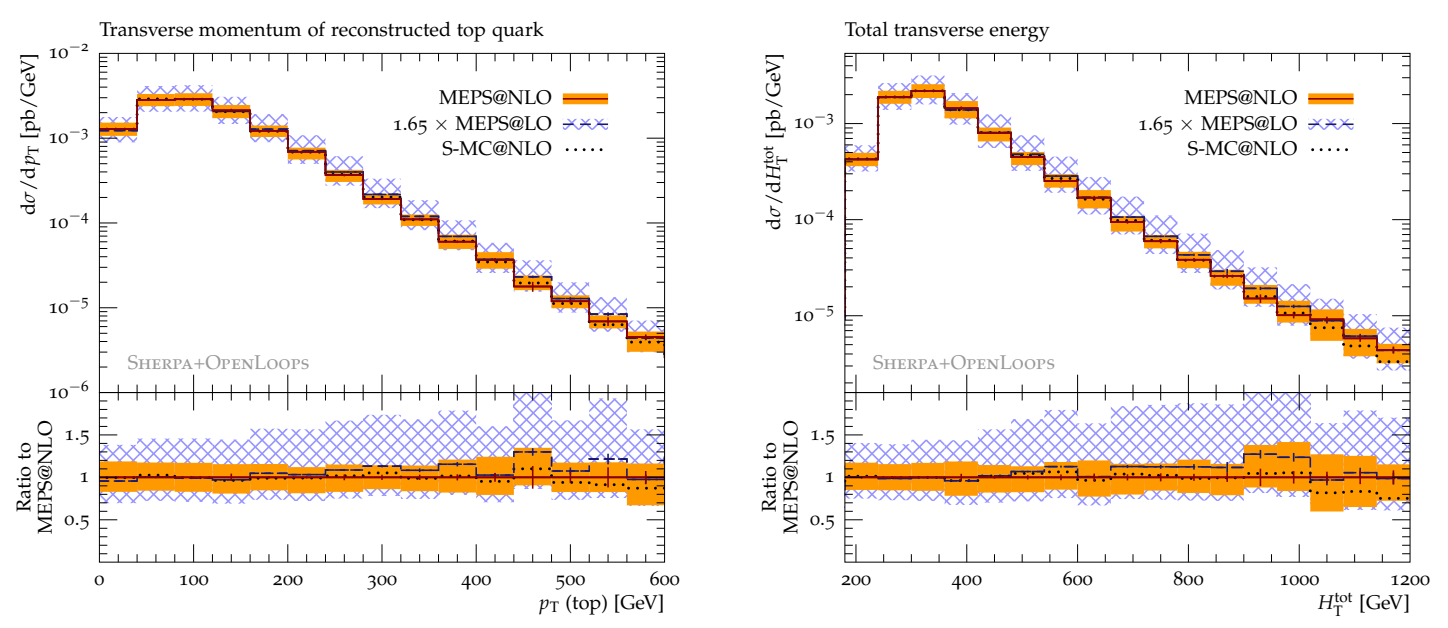

Figure 4: Transverse momentum of the reconstructed top quark (left) and total transverse energy (right). See Fig. 3 for details.

results rescaled with the inclusive $k$-factor 1.65 , and to a S-MC@ NLO sample. As compared to MEPs@Lo, the uncertainty of the inclusive MEPs@NLO cross section is reduced from $48 \%$ to $17 \%$, while that for events with at least one light-flavour jet of $p_{\mathrm{T}}>40 / 60 / 80 \mathrm{GeV}$ is reduced from $64 / 65 / 66 \%$ to $19 / 21 / 22 \%$ and with at least two jets from $79 / 81 / 83 \%$ to $21 / 27 / 34 \%$. The $Q_{\text {cut }}$ dependence of MEPS@NLO predictions is typically well below ten percent. A drastic reduction of the scale uncertainties is also achieved in jet transverse momentum distributions of the first and second jet. For the second jet, the scale dependence tends to increase for high $p_{\mathrm{T}}$, resulting from a large contribution from three-jet topologies which are described at leading order. Fig. 4 (left) shows the transverse momentum of the reconstructed top quark. Again we observe a strong reduction of uncertainties, particularly at larger transverse momenta. Finally, we analyse the total transverse energy, $H_{\mathrm{T}}^{\text {tot }}=\sum p_{\mathrm{T}, \mathrm{b}-\mathrm{jet}}+\sum p_{\mathrm{T}, 1-\mathrm{jet}}+\sum p_{\mathrm{T}, \text { lep }}+E_{\mathrm{T}}^{\text {miss }}$, of the full final state, where only light jets with $p_{\mathrm{T}}>40 \mathrm{GeV}$ are taken into account. This observable plays a key role in searches for new physics, and its high sensitivity to QCD radiation requires accurate modelling of multi-jet emissions. Fig. 4 (right) shows a strong reduction of perturbative uncertainties, especially in the high- $H_{\mathrm{T}}^{\text {tot }}$ region.

\section{Conclusions}

We applied SHERPA+OPENLOOPS to t⿱亠 production in association with a pair of massive bottom quarks and matched to the SHERPA parton shower, and tt merged with up to two jets using the MEPs@NLO method. Using massive b-quarks, the full phase space can be filled by NLO accurate t t $\mathrm{b} \overline{\mathrm{b}}$ matrix elements. Significant matching effects which exceed the Higgs signal in the $\mathrm{H} \rightarrow \mathrm{b} \overline{\mathrm{b}}$ decay channel are observed which can be attributed to splittings of gluons into collinear b-quark pairs. In $t \bar{t}+$ jets production a significant reduction of scale uncertainties is observed wrt. MEPs@LO and S-MC@NLO simulations, leading to considerably more accurate predictions especially for observables which are sensitive to multi-jet configurations. 


\section{Acknowledgments}

This work was supported by the SNSF, by the US Department of Energy under contract DE-AC02-76SF00515, and by the European Commission through the networks PITN-GA-2010264564, PITN-GA-2012-315877, and PITN-GA-2012-316704.

\section{References}

[1] F. Cascioli, P. Maierhöfer, and S. Pozzorini, Scattering Amplitudes with Open Loops, Phys. Rev. Lett. 108 (2012) 111601, [arXiv:1111.5206].

[2] G. Cullen, N. Greiner, G. Heinrich, G. Luisoni, P. Mastrolia, G. Ossola, T. Reiter, and F. Tramontano, Automated One-Loop Calculations with GoSam, Eur. Phys. J. C72 (2012) 1889, [arXiv:1111.2034].

[3] G. Bevilacqua, M. Czakon, M. Garzelli, A. van Hameren, A. Kardos, et al., HELAC-NLO, Comput. Phys. Commun. 184 (2013) 986-997, [arXiv:1110.1499].

[4] S. Actis, A. Denner, L. Hofer, A. Scharf, and S. Uccirati, Recursive generation of one-loop amplitudes in the Standard Model, JHEP 1304 (2013) 037, [arXiv:1211. 6316].

[5] J. Alwall, R. Frederix, S. Frixione, V. Hirschi, F. Maltoni, et al., The automated computation of tree-level and next-to-leading order differential cross sections, and their matching to parton shower simulations, arXiv:1405.0301.

[6] A. van Hameren, Multi-gluon one-loop amplitudes using tensor integrals, JHEP 0907 (2009) 088, [arXiv:0905.1005].

[7] A. Denner and S. Dittmaier, Scalar one-loop 4-point integrals, Nucl. Phys. B844 (2011) 199-242.

[8] A. Denner and S. Dittmaier, Reduction of one-loop tensor 5-point integrals, Nucl. Phys. B658 (2003) 175-202, [hep-ph/0212259].

[9] A. Denner and S. Dittmaier, Reduction schemes for one-loop tensor integrals, Nucl. Phys. B734 (2006) 62-115, [hep-ph/0509141].

[10] T. Gleisberg, S. Höche, F. Krauss, M. Schönherr, S. Schumann, F. Siegert, and J. Winter, Event generation with SHERPA1.1, JHEP 02 (2009) 007, [arXiv: 0811.4622 ].

[11] S. Höche, F. Krauss, M. Schönherr, and F. Siegert, A critical appraisal of $N L O+P S$ matching methods, JHEP 09 (2012) 049, [arXiv:1111.1220].

[12] S. Höche, F. Krauss, M. Schönherr, and F. Siegert, W+n-jet predictions with MC@ NLO in Sherpa, Phys. Rev. Lett. 110 (2013) 052001, [arXiv:1201.5882].

[13] S. Frixione and B. R. Webber, Matching NLO QCD computations and parton shower simulations, JHEP 06 (2002) 029, [hep-ph/ 0204244$].$

[14] T. Gehrmann, S. Höche, F. Krauss, M. Schönherr, and F. Siegert, NLO QCD matrix elements + parton showers in $e^{+} e^{-} \rightarrow$ hadrons, JHEP 1301 (2013) 144, [arXiv:1207.5031].

[15] S. Höche, F. Krauss, M. Schönherr, and F. Siegert, QCD matrix elements + parton showers: The NLO case, JHEP 1304 (2013) 027, [arXiv: 1207 . 5030].

[16] F. Cascioli, P. Maierhöfer, N. Moretti, S. Pozzorini, and F. Siegert, NLO matching for $t \bar{t} b \bar{b}$ production with massive b-quarks, arXiv:1309.5912. 
[17] S. Höche, F. Krauss, P. Maierhöfer, S. Pozzorini, M. Schönherr, et al., Next-to-leading order QCD predictions for top-quark pair production with up to two jets merged with a parton shower, arXiv: 1402.6293.

[18] A. Bredenstein, A. Denner, S. Dittmaier, and S. Pozzorini, NLO QCD corrections to pp $\rightarrow t$ anti-t $b$ anti-b + X at the LHC, Phys. Rev. Lett. 103 (2009) 012002, [arXiv: 0905.0110 ].

[19] A. Bredenstein, A. Denner, S. Dittmaier, and S. Pozzorini, NLO QCD corrections to top anti-top bottom anti-bottom production at the LHC: 2. full hadronic results, JHEP 1003 (2010) 021 , [arXiv:1001.4006].

[20] G. Bevilacqua, M. Czakon, C. Papadopoulos, R. Pittau, and M. Worek, Assault on the NLO wishlist: $p p \rightarrow t \bar{t} b \bar{b}$, JHEP 0909 (2009) 109, [arXiv : 0907 . 4723].

[21] M. Worek, On the next-to-leading order QCD K-factor for top $t \bar{t} b \bar{b}$ production at the TeVatron, JHEP 1202 (2012) 043, [arXiv:1112.4325].

[22] A. Kardos and Z. Trocsanyi, Hadroproduction of t anti-t pair with a b anti-b pair with PowHel, arXiv:1303.6291.

[23] A. D. Martin, W. J. Stirling, R. S. Thorne, and G. Watt, Parton distributions for the LHC, Eur. Phys. J. C63 (2009) 189-295, [arXiv:0901.0002].

[24] M. Cacciari, G. P. Salam, and G. Soyez, The Anti-k(t) jet clustering algorithm, JHEP 0804 (2008) 063, [arXiv:0802.1189].

[25] M. Czakon, P. Fiedler, and A. Mitov, The total top quark pair production cross-section at hadron colliders through $O\left(\alpha_{S}^{4}\right)$, Phys. Rev. Lett. 110 (2013) 252004, [arXiv:1303.6254].

[26] S. Dittmaier, P. Uwer, and S. Weinzierl, NLO QCD corrections to t anti-t + jet production at hadron colliders, Phys. Rev. Lett. 98 (2007) 262002, [hep-ph / 0703120 ].

[27] G. Bevilacqua, M. Czakon, C. Papadopoulos, and M. Worek, Dominant QCD Backgrounds in Higgs Boson Analyses at the LHC: A Study of pp $\rightarrow$ t anti-t +2 jets at Next-To-Leading Order, Phys. Rev. Lett. 104 (2010) 162002, [arXiv: 1002 . 4009 ].

[28] G. Bevilacqua, M. Czakon, C. Papadopoulos, and M. Worek, Hadronic top-quark pair production in association with two jets at Next-to-Leading Order QCD, Phys. Rev. D84 (2011) 114017, [arXiv:1108.2851].

[29] A. Kardos, C. Papadopoulos, and Z. Trocsanyi, Top quark pair production in association with a jet with NLO parton showering, Phys. Lett. B705 (2011) 76-81, [arXiv:1101.2672].

[30] S. Alioli, S.-O. Moch, and P. Uwer, Hadronic top-quark pair-production with one jet and parton showering, JHEP 1201 (2012) 137, [arXiv:1110.5251].

[31] R. Frederix and S. Frixione, Merging meets matching in MC@NLO, JHEP 1212 (2012) 061, [arXiv:1209.6215].

[32] S. Höche, J. Huang, G. Luisoni, M. Schönherr, and J. Winter, Zero and one jet combined NLO analysis of the top quark forward-backward asymmetry, Phys. Rev. D88 (2013) 014040, [arXiv:1306.2703].

[33] S. Schumann and F. Krauss, A parton shower algorithm based on Catani-Seymour dipole factorisation, JHEP 03 (2008) 038, [arXiv: 0709 . 1027].

[34] S. Höche, S. Schumann, and F. Siegert, Hard photon production and matrix-element parton-shower merging, Phys. Rev. D81 (2010) 034026, [arXiv: 0912.3501 ]. 